\title{
Perceptions From a Medical Student: A View of Addiction
}

\author{
Giuseppe Femia \\ Second-Year Medical Student, University of Sydney
}

Prior to medical school I believed addiction to be a selfinflicted condition. In fact, it seemed logical to me that among many other types of addictions, smokers were fully capable of quitting, and alcoholics too could cease drinking. In other words, I thought the addiction was psychological and could simply be overcome with a combination of education, willpower and dedication. This belief follows the theory of addiction termed 'the life process model of addiction', which has been supported by the notable psychologist, Stanton Peele (1985). In essence, the theory encompasses addiction, not as a disease, but rather a habitual response, source of gratification and security that one can derive from the particular substance. Both Peele and Szasz emphasised the individual's ability to overcome addiction by repairing relationships and personal willpower. I believe addiction to be a choice; an addict was therefore someone who consciously chooses a socially taboo substance over living a low-risk lifestyle. In another interpretation, The Myth of Addiction, Professor John Booth Davies (1992) asserts how individuals become inclined to take drugs because they want to and because it makes sense to them, given other choices available.

I began medical school in February 2006, where in week 9 we studied addiction - more specifically, addiction to heroin. I was amazed at the changes that occur in the human brain with respect to the reward pathway. I was aware of withdrawal symptoms, but not their effects on daily activities and the various ways in which they can cause addiction, and lead to serious depressive states or even death. Together with my misunderstanding of the process of reward pathways and the alteration of receptors using drugs, combined with the role of genetics and the detrimental effects attached, I was unable to separate my opinion from proven scientific research.

I now regard addiction as a disease with impairment of either neurochemical or behavioural processes. Unlike my previous assumptions, where I had a tendency to place all addiction subjects into one category, I understand that because of our privileged medical knowledge in the health-care profession, we have a responsibility to group people according to their illness. It should go without saying that not all individuals can be treated in the same manner; for example, some smokers respond well to NRT and give up cigarettes, while others require more invasive treatment such as receptor antagonists and atypical antidepressants. Upon reflection, I am quite disappointed with myself because I feel as though I perpetuated stereotypical perceptions of an already scrutinised group of individuals. Perhaps it is the presence of this prejudice and segregation that prevents those addicted from receiving proper attention and treatment. As a result, it is easy to understand why only $10 \%$ of individuals afflicted with an addiction actually receive treatment.

\section{References}

Peele, S. (1985). The meaning of addiction: An unconventional view. San Francisco: Jossey-Bass.

Davies, J.B. (1992). The myth of addiction. Amsterdam: Harwood Academic Publishers. 